\title{
Methane Abundance on Titan, Measured by the Space Telescope Imaging Spectrograph ${ }^{1}$
}

\author{
Mark T. Lemmon \\ Department of Atmospheric Sciences, Texas A\&M University, College Station, Texas 77843-3150 \\ E-mail: lemmon@tamu.edu \\ and \\ Peter H. Smith and Ralph D. Lorenz \\ Lunar and Planetary Laboratory, University of Arizona, Tucson, Arizona 85721-0092
}

Received November 21, 2001; revised July 25, 2002

\begin{abstract}
Although methane is the dominant absorber in Titan's reflection spectrum, the amount of methane in the atmosphere has only been determined to an order of magnitude. We analyzed spectra from the Space Telescope Imaging Spectrograph, looking at both a bright surface region (700-km radius) and a dark surface region. The difference between the spectra of the two regions is attributed to light that has scattered off the surface, and therefore made a roundtrip through all of Titan's methane. Considering only absorption, the shape of the difference spectrum provides an upper limit on methane abundance of $3.5 \mathrm{~km}$-am. Modeling the multiple scattering in the atmosphere further constrains the methane abundance to $2.63 \pm 0.17 \mathrm{~km}$-am. In the absence of supersaturation and with a simplified methane vertical profile, this corresponds to a surface methane-mole fraction near $3.8 \%$ and a relative humidity of 0.32 . With supersaturation near the tropopause, the surface methane mole fraction could be as low as $3 \%$. (c) 2002 Elsevier Science (USA)

Key Words: abundance, atmospheres; atmospheres, composition; satellites, atmospheres; spectrophotometry; Titan.
\end{abstract}

\section{INTRODUCTION}

The presence of methane in the atmosphere of Titan was discovered by Kuiper (1944). However, a precise measurement of the methane abundance has been difficult due to Titan's stratospheric haze, which obscures the surface from view within visible and near-infrared methane continuum regions and makes estimates based on methane bands model-dependent (Hunten et al. 1984). Recently, ground-based observations have shown that Titan has a light curve due to surface albedo features that are visible in methane continuum regions from 0.93 to $2.03 \mu \mathrm{m}$

\footnotetext{
${ }^{1}$ Based on observations made with the NASA/ESA Hubble Space Telescope obtained at the Space Telescope Science Institute, which is operated by the Association of Universities for Research in Astronomy, Inc., under NASA contract NAS 5-26555. These observations are associated with proposal \#7321.
}

(Lemmon et al. 1993, 1995; Griffith 1993; Coustenis et al. 1995). Observations with the Hubble Space Telescope have been used to map the surface features responsible for the light curve in many of the methane windows (Smith et al. 1996, Meier et al. 2000), and ground-based adaptive optics (Combes et al. 1997) and speckle (Gibbard et al. 1999) images have shown surface features at the longer wavelengths. Visibility of the surface indicates a two-way transmission of the light, which we can use to measure the methane abundance. A measurement of the total amount of methane in the atmosphere is important to the full interpretation of the images and light curves, because uncertainty in methane extinction contributes to a significant uncertainty in models of surface albedo (Lemmon 1994, Coustenis et al. 1995).

Much of our direct information about Titan's troposphere comes from the Voyager 1 radio occultation data set. Lindal et al. (1983) used this data set to determine temperature profiles for the ingress and egress of the occultation. However, due to uncertainty in methane abundance and profile, that determination is nonunique (Lindal et al. 1983). Lellouch et al. (1989) incorporated the existing measurements and chemical constraints to derive a family of possible profiles of methane mole fraction (MMF). Their nominal profile has a 1.5\% MMF in the stratosphere, increasing to $8 \%$ at the surface. Minimal and maximal profiles have from 0.5 to $3.4 \% \mathrm{MMF}$ in the stratosphere, and up to $21 \% \mathrm{MMF}$ at the surface, depending on the assumed argon abundance and temperature profile. A vertical integration of the methane profiles yields a total column abundance for methane ranging from 0.4 to $3.0 \mathrm{~km}-\mathrm{Am}$ for constant mole fractions of 0.5 to $3.4 \%$, and up to $6.9 \mathrm{~km}-\mathrm{Am}$ for the maximal profile of Lellouch et al. (1989). Lellouch et al. (1992) analyzed the region $9050-9160 \mathrm{~cm}^{-1}(1.10 \mu \mathrm{m})$ and determined that a total abundance of $4 \mathrm{~km}-\mathrm{Am}$ provided a better fit to the spectrum, but pointed out disagreements with earlier analyses of other bands.

While Lellouch et al. (1989) assumed that methane never exceeds its saturation value, Courtin et al. (1995) proposed that 
supersaturation may occur in the upper troposphere. They preferred a constant MMF in the range 2.6 to $4.5 \%$, which we determine to correspond to a total abundance of 2.3 to $4.0 \mathrm{~km}-$ Am. The analysis of Voyager UVS data by Strobel et al. (1992) also suggested a high MMF at the tropopause of 2.6 to $3.4 \%$. Samuelson et al. (1997) used Voyager IRIS data to derive the existence of a supersaturation region in the upper troposphere and determined that the surface MMF falls dramatically toward the poles (from $6 \%$ at the equator to $2 \%$ at $60^{\circ} \mathrm{N}$ or $\mathrm{S}$ ), as does the degree of supersaturation.

McKay et al. (1997) repeated the analysis of the radiooccultation data considering $\mathrm{CH}_{4}-\mathrm{N}_{2}$ condensation and the possibility of methane supersaturation. They found that the methane relative humidity at the surface could range from 0.08 to 1.0 , with values near 0.6 preferred. Given that most of the methane is likely to be in the bottom scale height $(20 \mathrm{~km})$ of the atmosphere, the total amount of methane is therefore only known to about an order of magnitude.

In this paper we consider a pair of spatially resolved spectra of Titan. Each samples the center of Titan's disk, one over the dark hemisphere and the other over the bright hemisphere. We use the difference between the center-of-disk spectra as a probe of the methane abundance. After describing the observations, we will discuss first a simple model and then a more complete model that we use to estimate the methane abundance. A detailed model of the distribution of scatterers in Titan's atmosphere is beyond the scope of this paper. We will then discuss implications of the measurement, given published constraints on the chemistry of Titan's troposphere.

\section{OBSERVATIONS}

\subsection{The Data Set}

The Space Telescope Imaging Spectrograph (STIS) was installed on the Hubble Space Telescope in 1997, and is described by Kimble et al. (1998). It is capable of obtaining spatially resolved spectra in the range 0.3 to $1.1 \mu \mathrm{m}$. The spatial axis (a slit across the object) has a resolution of 0.05 arcsec pixel ${ }^{-1}$. On 3 and 9 November 1997, we used STIS in its low-resolution

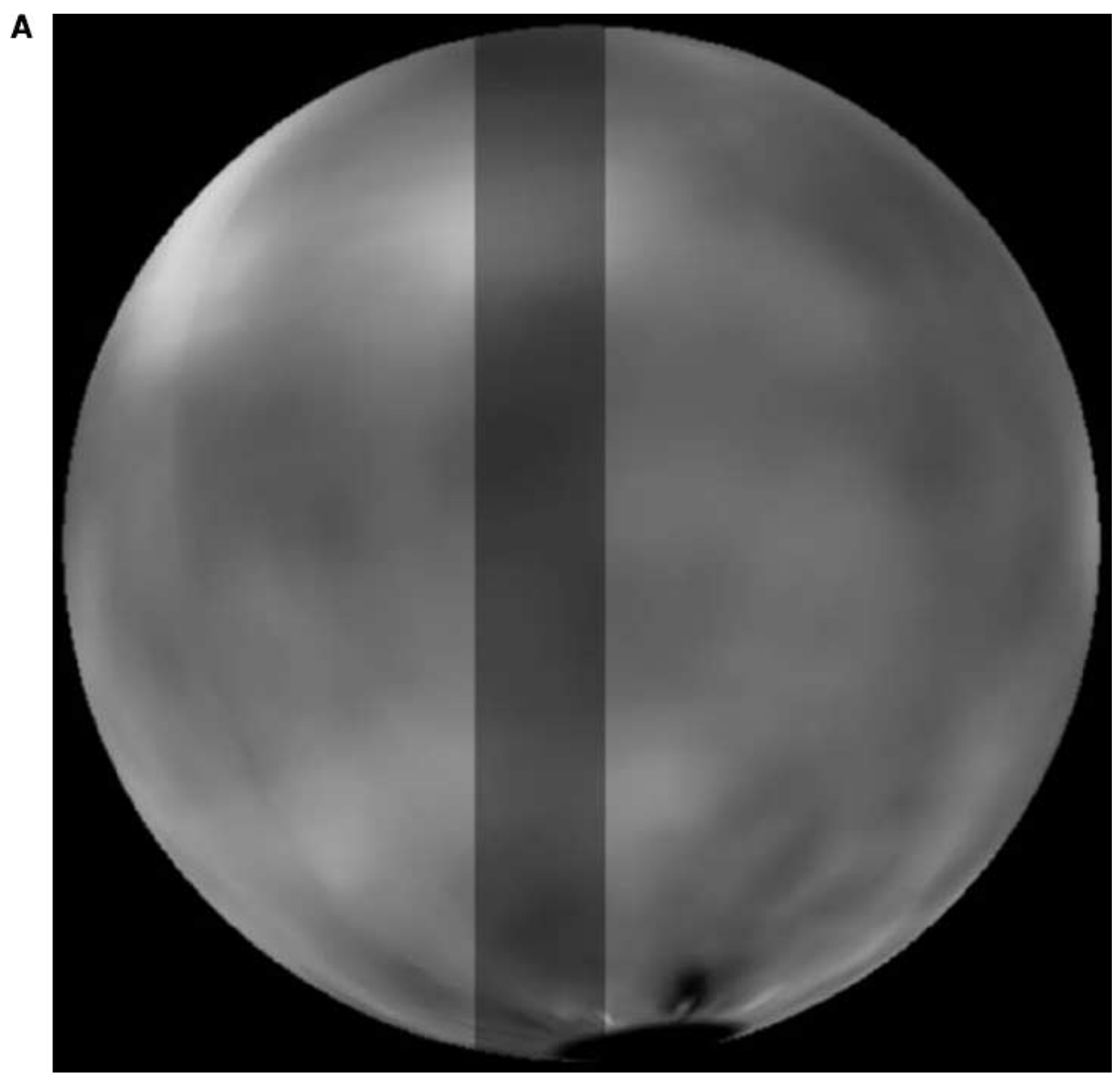

FIG. 1. Slit position projected onto the surface of Titan (maps taken from Lemmon et al., in preparation). The projected central slit is shown as the vertical darker stripe. (A) Observation of 11/3/1997, showing Titan's dark side (the bright features seen here may have been 1995 clouds). (B) Observation of 11/9/1997 showing Titan's bright feature, with the slit near its geographic eastern edge. The black area at the bottom of each image indicates the unmapped area around the south pole. 


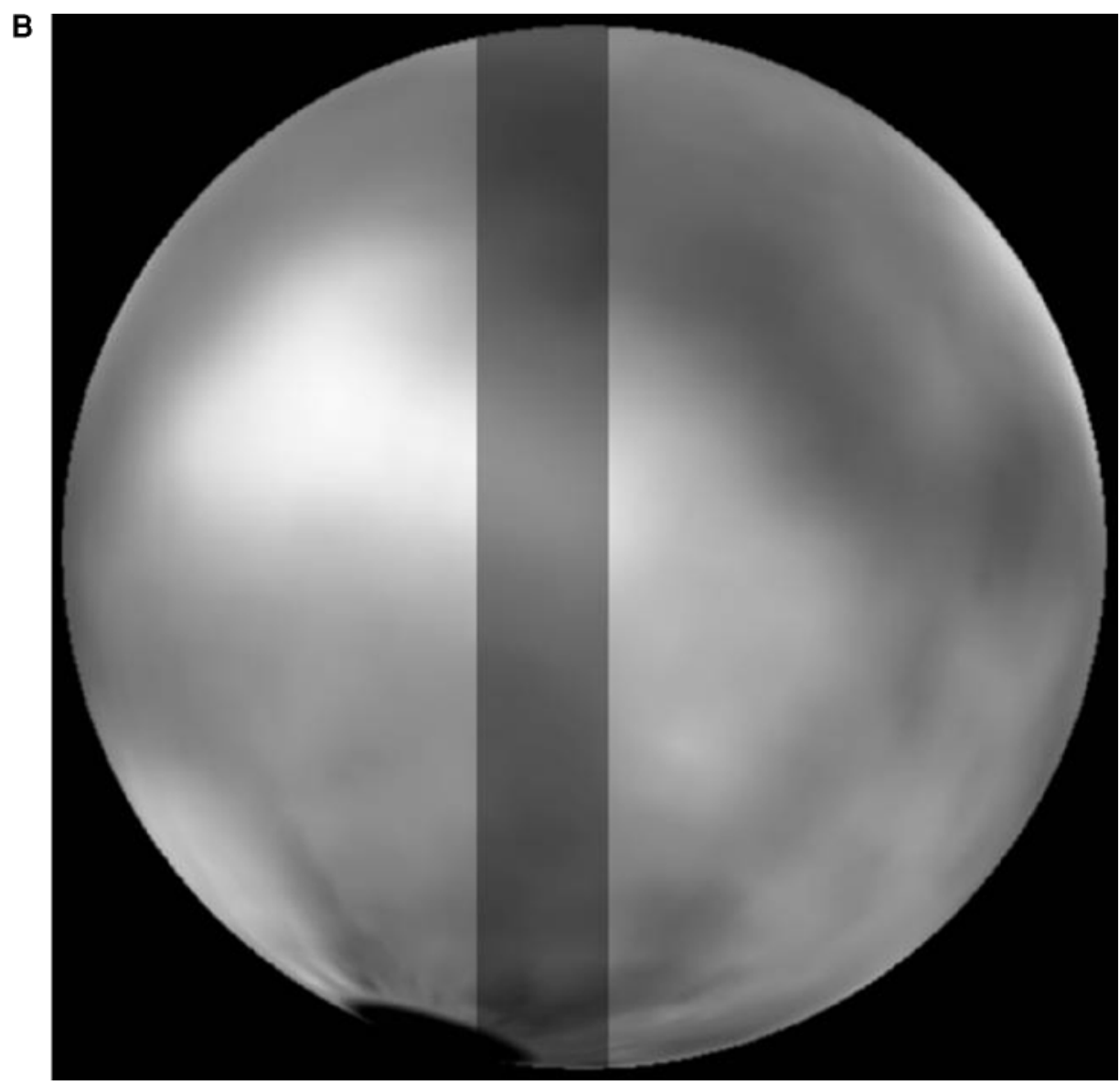

FIG. 1-Continued

(4.92 $\AA$ pixel ${ }^{-1}$ ) mode to obtain $0.30-1.0 \mu \mathrm{m}$ spectra of Titan (see Table I) as part of proposal GO 7321. Titan's angular size was 0.83 arcsec, giving some 16 pixels across the disk. We used the $52 \times 0.1$ aperture, which is an 0.1 -arcsec-wide slit. The spatial axis was aligned roughly north-south, but was not identical on the two days. For each observation, the slit was first aligned with the center-of-disk, and then was offset to the east by 0.1 and 0.2 arcsec to obtain spectra at different locations. For the first observation, Titan's longitude of central meridian (LCM) was $310^{\circ}$, and on the second it was $88^{\circ}$. The sub-Earth latitude during the observations was $9^{\circ} \mathrm{S}$, and the subsolar latitude was $11^{\circ} \mathrm{S}$.

\section{TABLE I}

Observational Parameters

\begin{tabular}{crccc}
\hline Date & LCM & Slit alignment & Phase angle $\left(^{\circ}\right)$ & Exposure time (s) \\
\hline 3 Nov 97 & 310 & -7.6 & 2.6 & 120.0 \\
9 Nov 97 & 88 & 16.6 & 3.2 & 120.0
\end{tabular}

Note. LCM is the longitude of the central meridian as seen from Earth; slit alignment is in degrees east of north.

\subsection{Calibration and Defringing}

The STIS data used here were processed through the onthe-fly-calibration pipeline in May 2000 using CALSTIS version 2.3. CALSTIS performs photometric, geometric, and wavelength calibrations. Nominal signal-to-noise was 50-150 for most of the spectra, except at wavelengths greater than $0.95 \mu \mathrm{m}$, where $S / N$ fell sharply due to the fall-off of CCD responsivity and Titan's low albedo, and in the center of the 890-nm methane band, where $S / N$ fell to $\sim 30$.

The calibrated spectrum is susceptible to long wavelength fringing, which is important at wavelengths longer than $0.7 \mu \mathrm{m}$ (Goudfrooij et al. 1997). In addition to the Titan spectra, we obtained a contemporaneous flat field to allow significant reduction of the fringing. The contemporaneous flat field is a calibration image using an on-board lamp and the $52 \times 0.2$ aperture. For each row (spectrum at one spatial position) in the resulting image, we fit a polynomial to the lamp intensity and divided the row by the fit. The residuals showed the fringing for the time and grating settings of our Titan observations. The fringes had a peak-to-peak amplitude of $\sim 25 \%$ near $0.95 \mu \mathrm{m}$. The fringing was low compared to the noise at wavelengths below $0.74 \mu \mathrm{m}$, so we set the fringing to unity at those wavelengths. 
We then divided the Titan image by the fringing image to obtain defringed spectra for each data set. This substantially reduces the fringing, but does not eliminate it due to slight wavelength shifts in the fringing pattern. Also, the fringing amplitude can vary from the observed value, as the fringe flat is created from a large, diffuse source, while Titan is diffuse but small.

For all spatial positions, the calibrated spectrum was divided by the solar spectrum, which was obtained by interpolating the spectrum of Neckel and Labs (1984)—similar to STIS in resolution-scaled to Titan's distance from the Sun (9.38 AU) to obtain an $I / F$ image. A few small artifacts remained due to the slight differences in resolution, but we judged these not to be a significant problem.

\subsection{Geometry}

The geometric calibration puts the centroid of Titan, as observed through a long-pass filter, on row 600 of the CCD. While this is supposed to be accurate to within $\sim 0.2$ pixels, Titan's centroid in the long-pass image is not the same as its geometric center due to a north-south brightness asymmetry. We found that the brightness asymmetry is very small at wavelengths near 0.54$0.57 \mu \mathrm{m}$ by plotting a north-to-south intensity profile over a south-to-north profile. For each image, we created a north-south profile by averaging all of the data for $0.54<\lambda<0.57 \mu \mathrm{m}$. By requiring the profile to be symmetric about the geometric center, we found the center to be 0.032 arcsec ( 0.6 pixels) north of the nominal center for the first data set, and 0.065 arcsec (1.3 pixels) north for the second. To derive the east-west position of the center we assumed that the relative offsets of 0.1 arcsec between spectra were accurate but the position of the first (nearcenter) image was uncertain. We determined the centroid of the half-maximum isophotes and found that the initial slit position was $0.043 \operatorname{arcsec}(0.86$ pixels) east of center for the first data day, and $0.039 \operatorname{arcsec}(0.77$ pixels) east of center for the second. We assume nominal uncertainties of 0.010 arcsec for the position of the center. During these observations, the phase angle increased from $2.6^{\circ}$ to $3.2^{\circ}$, and the subsolar point was 0.021 arcsec southwest $\left(241^{\circ}\right.$ east of north) of the disk center. For the first observation, the slit was aligned $7.6^{\circ}$ west of Titan's projected north pole; for the second it was $16.6^{\circ}$ east of Titan-north.

Table II summarizes the derived geometric parameters (neglecting very small offsets between the short- and longwavelength data sets). The orientation of the slits with respect to features on Titan (map projection based on Smith et al. (1996) and Lemmon et al., in preparation) is shown in Fig. 1. On the first day, the darker and more featureless side of Titan was visible. The features seen in the map may be cloud features observed in 1995 (Lemmon et al., in preparation), as they were not seen in 1994 by Smith et al. (1996) or in 1997 by Meier et al. (2000). For the second day, the large bright feature is visible and under the slit in all three positions. For further discussion, we will focus on the center-of-disk slit position only.
TABLE II

Slit Position

\begin{tabular}{lcccc}
\hline Date & $\begin{array}{c}\text { Data set } \\
(0.3-0.56 \mu \mathrm{m})\end{array}$ & $\begin{array}{c}\text { Data set } \\
(0.54-1.0 \mu \mathrm{m})\end{array}$ & $X$ & $Y$ \\
\hline 3 Nov 97 & o4dta3010 & o4dta3040 & $0.043^{\prime \prime}$ & 600.6 \\
3 Nov 97 & o4dta3020 & o4dta3050 & $0.143^{\prime \prime}$ & 600.6 \\
3 Nov 97 & o4dta3030 & o4dta3060 & $0.243^{\prime \prime}$ & 600.6 \\
9 Nov 97 & o4dta1010 & o4dta1040 & $0.039^{\prime \prime}$ & 601.3 \\
9 Nov 97 & o4dta1020 & o4dta1050 & $0.139^{\prime \prime}$ & 601.3 \\
9 Nov 97 & o4dta1030 & o4dta1060 & $0.239^{\prime \prime}$ & 601.3 \\
\hline
\end{tabular}

Note. $X=$ closest approach distance of slit center to Titan's center. $Y=$ row within image of Titan's center.

\subsection{Correction for Scattered Light}

The STIS CCD is susceptible to scattered light at longer wavelengths. This is a pronounced problem in our spectra of Titan. There is a significant amount of light tens of pixels from Titan. The off-disk spectrum rises from very small values at short wavelengths to become significant beyond $\sim 0.75 \mu \mathrm{m}$. The scattering brings light from nearby wavelengths to the same pixel: reduced contrast versions of Titan's methane bands can be seen, but the fringe spectrum cannot be seen. The off-disk spectrum falls exponentially with distance from Titan. Because it peaks at Titan, part of the Titan spectrum comes from this scattered light. To remove the scattered light, we fit a simple model: we use an exponential fall-off from a point source and convolve this with a line representing the size of Titan along the slit. We adjust the slope of the fall-off and the total amount of light to fit the data. Because the off-disk spectrum is degraded with respect to Titan's spectrum, we bin 25 pixels along the spectral dimension when deriving each fit. We then subtract the model of scattered light.

The above procedure removes the scattered light. Such light is undesirable since it does not have Titan's spectrum. However, Titan was originally the source of this light prior to scattering within the CCD. The instrumental scattering increases with wavelength, and we have corrected for the gain and not the loss due to scattered light, so we have not yet retrieved Titan's true spectrum. We correct the loss to scattered light by comparison with a set of WFPC2 data taken as part of the same proposal through the filters F336W, F439M, F547W, F588N, and F673N, and the methane band filters FQCH4N-B and FQCH4N-D at 0.619 and $0.89 \mu \mathrm{m}$ (see Lorenz et al. 1999a). In addition, we considered two images taken as part of the Meier et al. (2000) NICMOS data set through the F095N and F097N filters. For each filter and each spatial position along the STIS slit, we convolved the observed STIS spectrum with the filter's system spectral response. This was especially important for the $0.89-\mu \mathrm{m}$ methane band, as that filter has a small but significant leak of light near $0.84 \mu \mathrm{m}$, where Titan is much brighter. We found that at longer wavelengths the ratio of the image brightness to the STIS brightness increased exponentially with wavelength. 
We multiplied the short-wavelength data by a constant of 1.12 to force a match with the images; similarly, we multiplied the long-wavelength data by $\left(1+0.00956 e^{4.6054 \lambda}\right)$, where $\lambda$ is the wavelength in micrometers.

To check the result of the scattered light correction, we created a synthetic geometric albedo spectrum. We used the centerof-disk scan. For the northern and southern hemispheres separately, we evaluated the integral of $(2 \pi I r d r)$ from the center of disk $(r=0)$ to 20 pixels away (recall that Titan's apparent radius was 8 pixels), where the intensity had fallen below the noise. We divided this by the total flux from a Lambert-reflecting disk with a $2575-\mathrm{km}$ radius to obtain a geometric albedo. We compared this to the albedo of Karkoschka (1998), obtained in 1995. Differences between our synthesized albedo and the 1995 albedo were comparable to differences between Titan's 1993 and 1995 albedos reported in Karkoschka (1998). We also note that while the scattered light correction is critical to a detailed model of the spectrum, it has only a small effect on the methane measurement presented in this paper, as will be discussed below.

\subsection{The Difference Spectrum}

For each location on the disk (center, 0.1 arcsec east, and 0.2 arcsec east), the spectrum for $\mathrm{LCM} 310^{\circ}$ (dark side) was subtracted from the spectrum for LCM $88^{\circ}$ (bright feature). In order to do this, the spatial axis was expanded by a factor of 10 , and the two spectra were shifted along the spatial axis to align the observed northern and southern limbs across the entire image. The subtraction of bright minus dark was done for each pixel, and the result was restored to the original spatial scale. The recorded spectra include both light that has not interacted with the surface, scattering only in the atmosphere, and light that has scattered off the surface, which may have also scattered in the atmosphere. We assume there are no measurable east-west asymmetries within the atmosphere, so the subtraction effectively removes light that is scattered back to the observer without interacting with the surface. In addition, some of the light that interacts with the surface is removed, as we cannot assume the surface to be perfectly absorbing on the dark side. The subtraction spectrum is guaranteed to represent light that has scattered off something that is longitudinally heterogeneous. We will assume this to be the surface based on the known albedo feature and the longitudinal uniformity of the haze (note that the percentage variation is large compared to the methane clouds seen by Griffith et al. (1998, 2000), and the depth at which the variation occurs will turn out to be significantly deeper). So, the spectrum is the excess light from the bright side of the surface, as modified by atmospheric scattering and absorption. Figure 2 shows Titan's average spectrum and subtraction spectrum from the central four pixels. Within the four central pixels, the zenith angle and the solar zenith angle ( $\mu$ and $\left.\mu_{0}\right)$ are very near unity, so the light recorded by STIS passed through 2.03 airmasses on its way to the surface and back up to space (allowing for the pixel av-

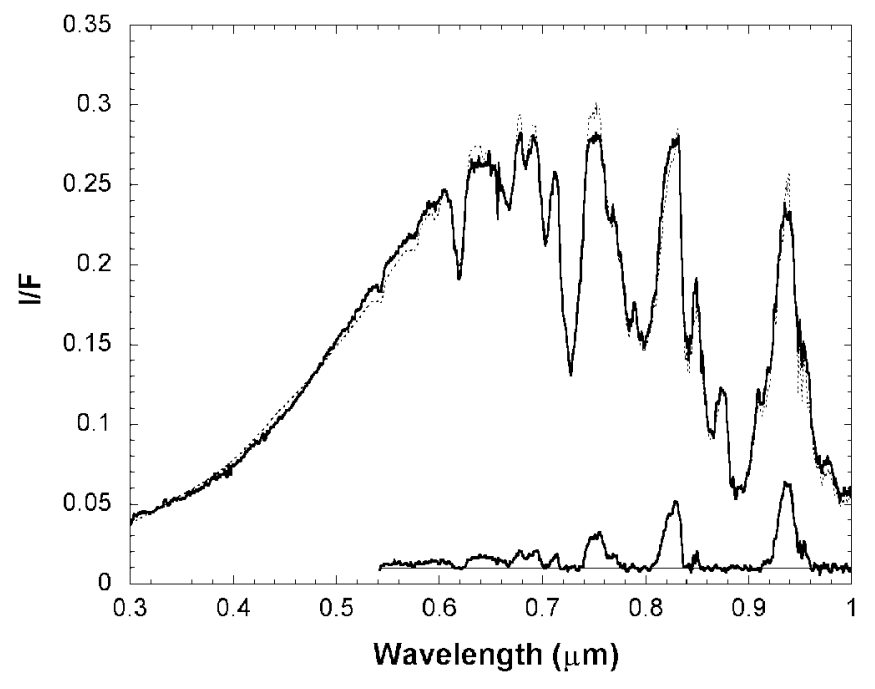

FIG. 2. Observed center-of-disk spectrum. The average (bright + dark) $/ 2$ spectrum is shown (upper solid line) with a simple model (dashed line, see Section 3.3). The difference spectrum (bright - dark), including surface light, is shown (lower solid line) offset by +0.01 .

eraging and the instrument point-spread function). In the next section, we discuss the role of multiple scattering on the effective path length the light passes through.

\section{DISCUSSION}

\subsection{Modeling the Methane Spectrum}

Titan's spectrum contains a series of methane bands of varying strengths. There have been several measurements of the abundance of methane in Titan's atmosphere based on the band strengths seen in Titan's visible geometric albedo (e.g., Trafton 1975; Lutz et al. 1976, 1982). It was noted that more methane could be seen at longer wavelengths, indicating that scattering in the atmosphere was important and suggesting that the atmosphere might be optically thick in the visible. We now know that the haze is optically thick at all wavelengths below $1 \mu \mathrm{m}$, but becomes increasingly transparent at longer wavelengths, in part because the aerosols are absorbing below $0.5 \mu \mathrm{m}$, but scattering above $0.7 \mu \mathrm{m}$ (see, for example, the models of Griffith $e t$ al. (1991), the observations of the surface by Lemmon et al. (1993), and the recent review by McKay et al. (2001)).

In order to interpret the spectrum, we used the absorption coefficients of Karkoschka (1998). These coefficients give absorption per kilometer-amagat of methane at the same spectral resolution as our STIS spectra. They were derived to have the same band strengths for each methane band as measured in the laboratory at room temperature (by Benner (1979)); the profile of the band was adjusted to match the spectra of Jupiter, Saturn, Uranus, Neptune, and Titan. The result is a cold-temperature methane spectrum, appropriate for modeling observations like ours. While Titan's geometric albedo was considered in the derivation of the spectra, Karkoschka (1998) found insignificant 
variation in results from each planet (with exceptions noted below). In any case, the analysis omitted any assumptions about the atmospheric structure, and relied only on the assumption that the atmospheric scattering was smoothly varying with wavelength, rather than exhibiting bands (other atmospheric gases were accounted for).

We use this set of absorption coefficients advisedly. In this approach, we ignore the individual spectral lines that make up the bands. For most of the spectrum we consider, this is appropriate. Lutz et al. (1976) presented curves of growth for most of the visible methane bands and applied these to studies of the outer planets and Titan. They found that each band was described by a single, pressure-independent curve of growth. This implied that, quite unlike the case for longer wavelength methane bands, discrete line structure was absent (at any resolution for pressures like those where observable methane absorption happens). Lutz et al. (1976) concluded that these bands were ideal for methane abundance determinations, precisely because they are pressure independent. Lutz et al. (1982) extended the analysis to the red and near infrared methane bands (to $0.725 \mu \mathrm{m}$ ). As in their earlier analysis, they found that a single pressure-independent curve of growth described absorption in all of the methane bands they considered. They also discuss the temperature independence of the band strength noted in the analysis of Karkoschka (1998). As a check, we have also used the Karkoschka absorption coefficients to compute a synthetic curve of growth for several of the methane bands. We found that this curve of growth well matched that presented by Lutz et al. (1982).

Karkoschka (1998) found that, for most of the spectrum, the same set of absorption coefficients worked for each of the outer planets and Titan. For a few parts of the longer wavelength bands, however, there was a disagreement between Jupiter and the other cases that he attributed to temperature dependence. Also, at the longer wavelengths the detailed band structure becomes important (e.g., methane lines can be seen in the $3 v_{3}$ band at $1.1 \mu \mathrm{m}$, and analysis of that band depends on both abundance and pressure). In the determination to follow, we will note any measurements that depend on the discrepant wavelengths. While these are likely to be accurate for Titan, they will be given less weight than the purely pressure-independent bands.

\subsection{Reflecting-Layer Model}

We chose to begin with a simple model of the spectrum to measure the methane abundance. We modeled the spectrum as that of a reflecting layer of arbitrary (but smoothly varying) brightness under some amount of methane. We followed the procedure of choosing an array of possible two-way methane column abundances from 0 to $15 \mathrm{~km}$-am and testing each one $\left(N_{\text {test }}\right)$ as follows. A methane continuum was defined as the set of wavelengths with absorption $\left(k_{\mathrm{CH}_{4}} \times N_{\text {test }}\right)<0.2$, where the methane absorption coefficient $k_{\mathrm{CH}_{4}}$ is taken from Karkoschka (1998). The small amount of methane absorption in the "continuum" was removed by dividing out the model absorption using the test abundance. A polynomial in wavelength was then fitted to

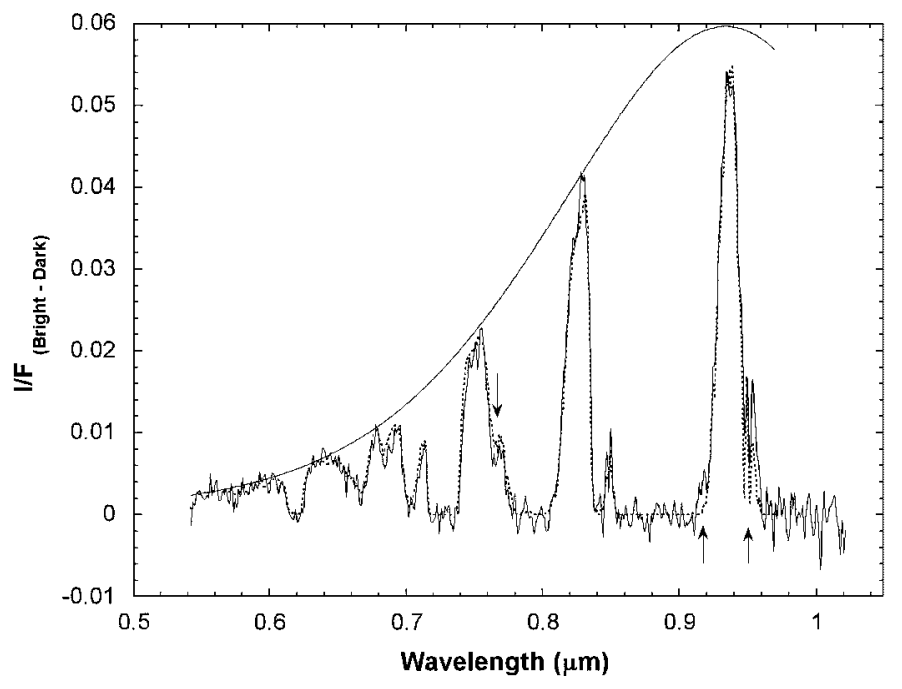

FIG. 3. Difference spectrum. The center-of-disk surface (bright - dark) spectrum (solid line) is compared to a continuum fit (upper light solid line) and a model spectrum including methane absorption (heavy dashed line). Arrows indicate especially poor fits.

the continuum points. The polynomial fit was evaluated at each wavelength to provide a continuum spectrum (see Fig. 3), and that spectrum was multiplied by $e^{\left(-k_{\mathrm{CH}_{4}} \times N_{\text {test }}\right)}$ at all wavelengths to determine a model spectrum. The residuals (observation model) were computed, $\chi^{2}$ was determined, and a probability $P\left(\chi^{2}\right)$ was computed. The best fit, shown in Fig. 3, is for a total path abundance of $7.20 \mathrm{~km}-\mathrm{Am}$, which corresponds to a one-way column abundance of $3.55 \mathrm{~km}$-Am given the average airmass of 2.03. We used the Lellouch et al. (1989) atmospheric profile to convert abundance into an estimated surface MMF. Following this profile, we assumed the stratospheric MMF to be $1.5 \%$; the tropospheric MMF was held equal to the saturation value in the upper troposphere, and equal to the surface MMF in the lower troposphere. The transition between the "upper" and "lower" troposphere, in this case, is the altitude where the saturation vapor profile is equal to the surface MMF: the MMF is not allowed to climb above this value lower in the troposphere. We found $3.55 \mathrm{~km}-$ Am to correspond to a surface MMF of $7.0 \%$ (and a relative humidity at the surface of 0.57). Such a simple approach is not guaranteed to fit the constraints employed by Lellouch et al. (1989), but is used only to provide a rough translation of our derived column abundance to a surface MMF. While our derived surface relative humidity value closely corresponds to the preferred value of McKay et al. (1997), there are important problems with this simple analysis.

Uncertainty in the absolute responsivity of the instrument is approximately $10 \%$, but is not important to this measurement. The absolute responsivity, the correction for scattered light, the surface albedo, and the haze transparency all contribute to the continuum spectrum, but only the shape of the methane bands contributes to the methane determination. For this reason, the smoothly varying scattered light correction affects the shape 
TABLE III

Reflecting-Layer Model Methane Abundances

\begin{tabular}{lcc}
\hline Wavelength range $(\mu \mathrm{m})$ & Abundance $(\mathrm{km}-\mathrm{am})^{a}$ & $\sigma(\mathrm{km}-\mathrm{am})$ \\
\hline & \multicolumn{2}{c}{ Bands } \\
$0.60-0.64$ & 5.52 & \\
$0.635-0.678$ & 3.60 & 0.89 \\
$0.678-0.75$ & 5.17 & 0.34 \\
$0.75-0.83$ & 3.69 & 0.34 \\
$0.83-0.935$ & 3.05 & 0.25 \\
$0.935-1.00$ & 2.61 & 0.12 \\
& Windows & 0.17 \\
$0.54-0.625$ & 4.63 & 1.13 \\
$0.61-0.73$ & 4.43 & 0.30 \\
$0.72-0.80$ & 4.68 & 0.30 \\
$0.795-0.90$ & 3.20 & 0.15 \\
$0.87-0.97$ & 2.86 & 0.12 \\
\hline
\end{tabular}

\footnotetext{
${ }^{a}$ Column abundance, or approximately the total path abundance divided by 2.03 airmasses.
}

of the continuum, but does not have a significant effect on the methane measurement. Much more importantly, the fit shown in Fig. 3 is not a statistically good fit as determined by reduced $\chi^{2}$ : there are systematic errors in many of the methane bands. Arrows denote some of the errors (note that the arrow near $0.92 \mu \mathrm{m}$ also corresponds to a wavelength identified as suspect by Karkoschka (1998)).

The statistically poor fit is because we have so far omitted some of the important physics of the problem, as we now demonstrate. We broke the spectrum into pieces, as follows. First, we isolated individual methane bands, defined as the entire region between adjacent continua (see Table III). Second, we isolated the continua (between band midpoints, also in the table). We found the best-fit methane abundance for each band or continuum following the same procedure described above; these fits are reported in Table III and shown graphically in Fig. 4. Each individual fit was quite good in a $\chi^{2}$ sense and meaningful uncertainties could be determined.

Note the strong wavelength dependence in Fig. 4: the derived methane abundance in the reflecting-layer model is a function of wavelength. The reflecting-layer model must therefore be missing something important in its description of Titan. There are two observations we can bring to bear. First, in the reflecting-layer model, as the haze becomes more transparent at longer wavelengths, the apparent methane abundance decreases. Second, this trend is exactly opposite the trend seen when observing Titan's geometric albedo (e.g., Lutz et al. (1982)). The spectrum of an atmosphere containing small scatterers mixed with an absorbing gas shows greater absorption at longer wavelength, because the more transparent hazes allow light to penetrate more deeply. In the present observations, we are looking at light that penetrates to a specific level and is reflected by the surface (not the haze). Where the haze is transparent, the path is simply in and out. Where the haze is more optically thick, there can be multiple scattering, increasing the actual path length the photons tra- verse. Thus, we observe less absorption at longer wavelengths. In either case, multiple scattering is important and must be modeled to derive a wavelength-independent methane abundance. This will be done in the next section.

We note that the determinations using individual methane bands (except for the longest wavelength band, where only half of the band was observed) are equivalent to a curve-of-growth technique. The method used here has the advantage that it can be applied at these large path lengths, where many of the bands blend into one another, through the use of spectrally resolved absorption coefficients. Without making a more complicated model, we can derive an upper limit to the methane abundance. The flanks of the methane window at $0.938 \mu \mathrm{m}$ have methane coefficients subject to a temperature-related uncertainty according to Karkoschka (1998). The methane window at $0.83 \mu \mathrm{m}$ does not have this problem, so we regard it as being a more trustworthy probe of the methane abundance. The methane column abundance measured using the $0.83-\mu \mathrm{m}$ window is $3.20 \pm$ $0.15 \mathrm{~km}-\mathrm{am}$; thus, the $2-\sigma$ upper limit on methane column abundance is $3.50 \mathrm{~km}-\mathrm{am}$. If we allow the use of the longest wavelength data in Table III, the upper limit becomes $3.0 \mathrm{~km}-\mathrm{am}$, but we are skeptical of the longest wavelength window based on the discussion of temperature-related uncertainty in Karkoschka (1998).

The north-south orientation of the slit allowed us to check for latitudinal variations. We repeated the analysis for each of the bands at all spatial positions along the slit. After allowing for the longer path length due to purely geometric considerations, we found no statistically significant variation. The data suggest, but do not require, lower methane abundances near both poles. We did not account for the fact that the instrument point-spread function scatters light from different latitudes into

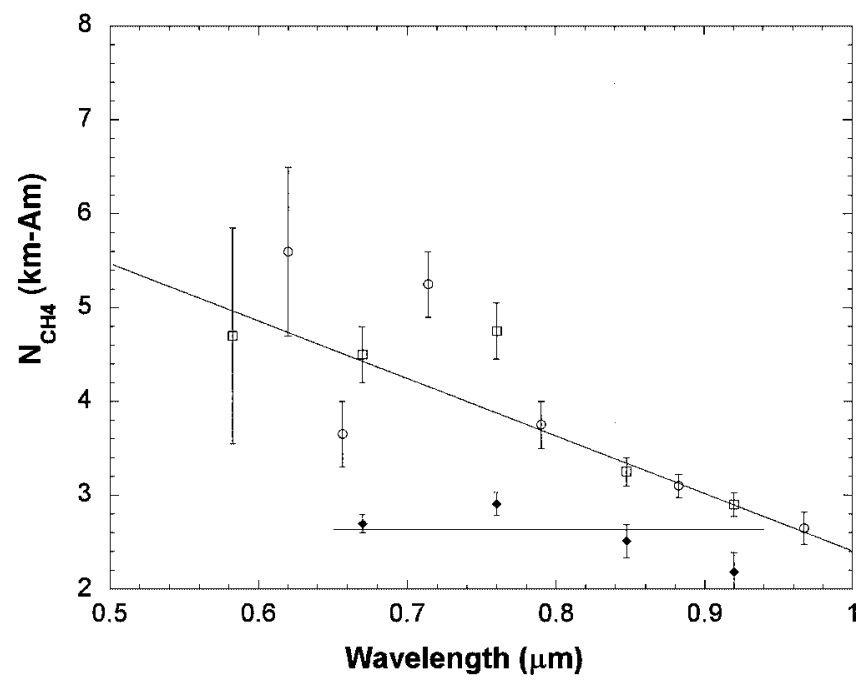

FIG. 4. Methane abundance by band $(\bigcirc)$ and continuum ( $\square$ ). The abundance is converted into a column abundance by dividing by the two-way transmission. A linear fit is shown for reference; clearly multiple scattering is important below $0.9 \mu \mathrm{m}$. Also shown are results of a multiple-scattering model $(\diamond ;$ see Section 3.3) with a line indicating the best-fit measurement. 
the same pixel. A preliminary analysis indicated that this effect reduces the statistical significance further. Therefore, we have a good constraint on near-equatorial methane abundance, but will not further investigate latitudinal variations in this work.

\subsection{Multiple Scattering}

To evaluate the effect of multiple scattering, we derived a simple model of Titan's haze. The model is "simple" in that it fits Titan's reflection spectrum (Fig. 2) well, with a small number of free parameters. We assumed all particles to be spherical (Mie scattering) and to have a refractive index corresponding to tholin (Khare et al. 1984). Others have suggested darker haze particles (e.g., McKay et al. 1989, McGrath et al. 1998). Because absorption by the aerosols is already so low in the spectral region considered here, an increase in absorption by tens of percent would have little impact on our results. We also assumed a gamma size distribution as given by Hansen and Travis (1974) with a width of $b=0.2$. We assumed a thin layer $\left(\tau_{0.8 \mu \mathrm{m}}=\right.$ 0.058 ) of $a=0.3-\mu \mathrm{m}$ spheres at $300 \mathrm{~km}$ following the observations of Karkoschka and Lorenz (1997). We added a stratospheric haze with $a=0.1-\mu \mathrm{m}$ spheres to fit the violet and $0.89-\mu \mathrm{m}$ data. Thus the stratosphere is somewhat similar to the model of large spheres above small spheres discussed by Tomasko and Smith (1982). We did not model the haze as aggregate aerosols (e.g., West and Smith 1991, Rannou et al. 1995) because the data set did not require the additional assumptions and complication. Previous haze models have not determined a haze structure specific to near-equatorial regions just after southern spring equinox. The details of the stratospheric haze structure are poorly constrained by the near-infrared data, so a low vertical resolution was used. The minimum structure we were forced to incorporate was to put some of the haze $\left(\tau_{0.8 \mu \mathrm{m}}=0.23\right)$ above all of the $0.89-\mu \mathrm{m}$ methane absorption, between 150 and $300 \mathrm{~km}$; and more haze $\left(\tau_{0.8 \mu \mathrm{m}}=0.36\right)$ low enough to show structure in the center of the band (with the absorption coefficient, $k>20 \mathrm{~km}-\mathrm{am}^{-1}$ ), nominally between 90 and $150 \mathrm{~km}$. We emphasize that details of this structure are not well constrained.

We added two tropospheric haze layers with $a=0.15-\mu \mathrm{m}$ spheres to fit the shape of the 0.7- to $1.0-\mu \mathrm{m}$ methane bands (Fig. 2). The size and shape of tropospheric scatterers are not well constrained by previous data sets, and we find a sphericalparticle model adequate for this task. The particles are assumed to be larger than the stratospheric particles based on the observation of increasing particle size with depth from Tomasko and Smith (1982). Models with $a=0.12,0.15$, and $0.20 \mu \mathrm{m}$ were tested; the $0.15-\mu \mathrm{m}$ particles provided the best fit. The sensitivity to size comes from the increasing transparency of the haze with increasing wavelength; larger particles become transparent more slowly than smaller particles. Spectral regions of strong methane absorption $\left(3>k>10 \mathrm{~km}-\mathrm{am}^{-1}\right)$ were used to constrain the altitude and opacity of a single tropospheric haze layer $\left(\tau_{0.8 \mu \mathrm{m}}=0.40\right.$ at $39 \mathrm{~km}$ altitude). Spectral regions of weaker methane absorption $\left(1>k>2 \mathrm{~km}-\mathrm{am}^{-1}\right)$ were used to constrain the altitude and opacity of a second tropospheric haze layer $\left(\tau_{0.8 \mu \mathrm{m}}=0.23\right.$ at $25 \mathrm{~km}$ altitude). The total haze opacity in the model is $\tau_{0.8 \mu \mathrm{m}}=1.3$, with $\tau_{0.64 \mu \mathrm{m}}=2.5$.

The model presented here is nonunique; that is, the observations do not require the specific distinct layers (as opposed to a gradient in density or a more complicated profile). The two tropospheric haze layers could be sufficiently spread out that they merge; either one of them could be replaced by more complicated structure (e.g., the 39-km-high layer could be replaced by a 30-km-high layer and a 55-km-high layer, leaving the upper troposphere free of scatterers). The virtue of the model is that it is simple and incorporates a good estimate of the effects of multiple scattering. The model is robust against errors in the assumed absorption coefficient of the haze. In the case of darker haze particles, there is less multiple scattering due to the increase in aerosol absorption. However, to the extent the particles are darker, observed brightness in the core of the methane bands requires more haze (and thus more multiple scattering). To first order, these effects cancel. Thus, for the low-absorption regime considered here, the exact aerosol absorption coefficient is not an important source of uncertainty.

We evaluated this model for varying ground reflectivities to test whether multiple scattering involving the surface is important. For each continuum, we determined a difference in ground reflectivity (bright minus dark) and methane column abundance, first assuming the average ground reflectivity ([bright + dark]/2) was 0.15 , then assuming it to be 0.25 . We found the same results for both cases, so we constrain only the difference in ground reflectivity between the bright and dark areas, not the absolute reflectivity. Figure 4 shows the best-fit methane column abundance for four windows. The determined abundance and difference in ground reflectivity in each window is shown in Table IV. For much larger ground reflectivities, the differences would be slightly smaller; for a bright ground reflectivity below 0.4 , the results in Table IV are sufficiently general. Ignoring the longest wavelength window (as potentially unreliable due to temperature dependence and the possibility of residual fringes), we find an average abundance of $2.63 \pm 0.17 \mathrm{~km}-\mathrm{am}(1-\sigma)$, and no evidence of residual systematic wavelength dependence.

\section{TABLE IV}

\section{Multiple-Scattering Model Methane Abundances and Difference} in Ground Reflectivity

\begin{tabular}{lccr} 
Wavelength range $(\mu \mathrm{m})$ & Abundance $(\mathrm{km}-\mathrm{am})^{a}$ & $\sigma(\mathrm{km}-\mathrm{am})$ & $\Delta \mathrm{GR}$ \\
\hline & Windows $^{b}$ & & \\
$0.61-0.73$ & 2.7 & 0.1 & 0.055 \\
$0.72-0.80$ & 2.91 & 0.18 & 0.062 \\
$0.795-0.90$ & 2.51 & 0.21 & 0.091 \\
$0.87-0.97$ & 2.18 & & \\
& & & \\
\hline & & & \\
${ }^{a}$ Column abundance. & &
\end{tabular}




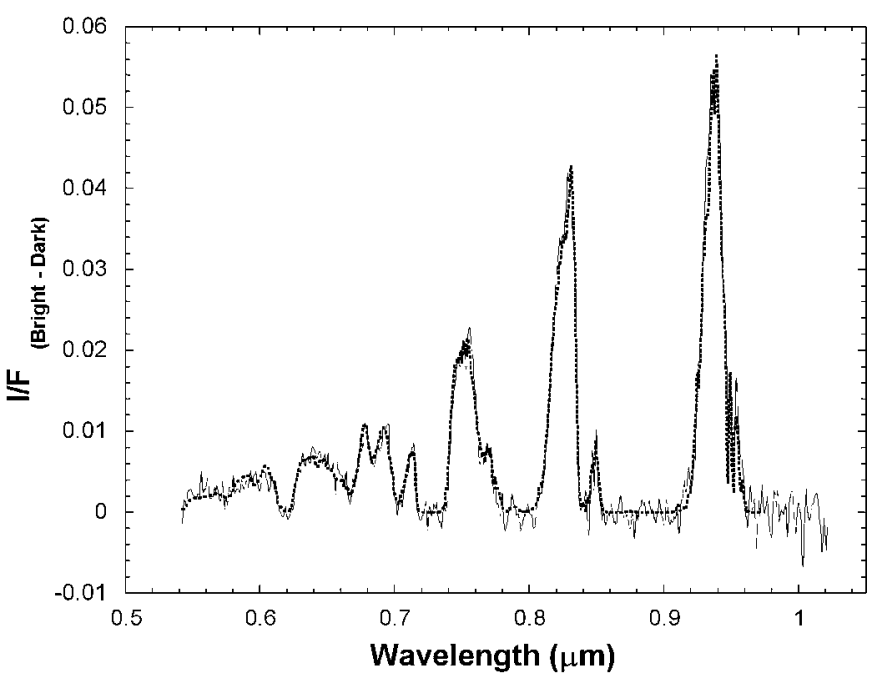

FIG. 5. Difference spectrum. Similar to that described in the legend to Fig. 3, but for the multiple-scattering model. Note the improved fit compared to Fig. 3 near $0.69,0.77$, and $0.95 \mu \mathrm{m}$.

Figure 5 shows the observed and model difference spectra using this abundance.

To test the model dependence of this result, we found that the difference between the simple reflecting-layer model result and a multiple-scattering model result was similar when using the fractal aggregate model of Lemmon (1994) and Tomasko et al. (1997) instead of the Mie scattering model. The fractal aggregate model incorporates about $\tau=0.5$ (wavelength independent) of scatterers evenly distributed between the surface and $90 \mathrm{~km}$ altitude. This opacity provides similar multiple scattering to the three layers used in the present model, highlighting the nonuniqueness of the vertical distribution of scatterers.

Again assuming a stratospheric MMF of $1.5 \%$ and applying the simplified methane profile introduced in Section 3.2, we find the surface MMF to be $3.8 \%$, corresponding to a relative humidity of $32 \%$. This is significantly lower than the preferred value of $60 \%$ from McKay et al. (1997). The low methane abundance is consistent with a low absolute reflectivity for the surface. For Titan to have its observed surface temperature of $94 \mathrm{~K}$, some $12 \mathrm{~K}$ higher than the effective temperature, requires a strong greenhouse effect (e.g., McKay et al. 1989) driven principally by $\mathrm{N}_{2}$. Although downwelling thermal radiation from the warm haze affects the heat balance, the high surface temperature requires some sunlight to be absorbed at the surface. Analytic approximations (Lorenz et al. 1999b) to the McKay et al. numerical radiative-convective model suggest that if Titan's average surface reflectivity is 0.4 , a methane profile with a surface relative humidity of about $60 \%$ is needed to provide enough greenhouse warming to produce the observed surface temperature. On the other hand, if the average surface reflectivity is only 0.1 , more sunlight is absorbed at the surface and hence only a weaker greenhouse effect is needed, with a relative humidity near $40 \%$. These models have many assumed parameters, of course, but are a largely independent constraint: the 0.1 surface reflectivity and $40 \%$ relative humidity parameter set is one that satisfies the climate model and is broadly consistent with the methane determination in the present paper.

While Lutz et al. (1982) found the 0.6825- and 0.7050- $\mu \mathrm{m}$ methane bands to be problematic, we do not. These are the weakest of the near-infrared bands (Lutz et al. did not consider the band at $0.66 \mu \mathrm{m}$ ) and are not saturated in the difference spectrum (see Fig. 5). Thus, these bands are fully sensitive to the surface. Lutz et al. (1982) measured methane path abundances of 2.81 and $1.09 \mathrm{~km}$-am in these two bands using whole-disk observations; the former measurement is similar to our estimate of the total methane path abundance for this wavelength region from Table IV. They estimated much lower abundances from the stronger bands, which were dominated by photon paths that never left the stratosphere. That is, in the absence of methane absorption, there would be a distribution of photon paths such that many were scattered from the upper stratosphere, many penetrate lower, and many make it to the surface and back. At longer wavelengths, the distribution is shifted lower in the atmosphere. Stronger methane bands select those photons that do not penetrate deeply even in the absence of absorption; weaker methane bands sense all of the path lengths. The present result, showing that methane absorption and multiple scattering are just weak enough that the surface is sensed between 0.63 and $0.7 \mu \mathrm{m}$, cannot be considered surprising, as Titan's bright surface feature was seen by Smith et al. (1996) using a 0.67- $\mu$ m filter.

\section{CONCLUSIONS}

We have measured the column abundance of methane in Titan's equatorial regions. Use of a multiple-scattering model allowed us to determine the methane column abundance was $2.63 \pm 0.17 \mathrm{~km}-\mathrm{am}$. If we have overestimated the effect of multiple scattering, the methane column abundance must still be less than $3.5 \mathrm{~km}$-am. Our determination applies specifically to the equatorial regions in 1997: we have assumed no eastwest asymmetries. With this data set, we were unable to provide a useful constraint on the presence of latitudinal asymmetries. Further data would be quite useful for tracking possible seasonal variation.

We measure the bulk methane abundance through the atmosphere, with no information on vertical distribution. By assuming the Lellouch et al. (1989) distribution, but allowing the surface MMF to vary, we find that the mole fraction near the surface is $3.8 \%$. This corresponds to a methane relative humidity of $32 \%$ at the surface, approximately half of the preferred value from McKay et al. (1997). If there are important variations in the methane humidity at the surface, for instance due to an increased concentration of ethane-methane lakes in darker areas or a significant elevation difference, then the measured humidity refers more to the bright side. If, on the other hand, the bright area is an orographic methane fog, multiple scattering within the fog combined with hiding of methane below the fog may cause a significant misestimation of the actual abundance at 
the surface. However, no particular evidence for such effects is seen.

The surface MMF may be lower if there is more methane in the stratosphere or upper troposphere than we have assumed. Our measured abundance is consistent with a constant mole fraction of $3 \%$. Such a profile, while not particularly physical given that methane has a surface source and a stratospheric sink, is consistent with the constraint of Strobel et al. (1992) that the MMF at the tropopause is $>2.6 \%$. If the stratospheric MMF is a constant $2.6 \%$, and methane were supersaturated at that mole fraction in the upper troposphere, a surface MMF of $3.3 \%$ would yield our observed abundance. In these cases, the presence of supersaturation and high levels of stratospheric methane requires the surface methane humidity to be as low as $25-27 \%$.

Each of the methane profiles discussed here is a simple perturbation to an established model. A future extension of this work would be to combine the present result, a constraint on methane column abundance, with the constraints derived by Strobel et al. (1992) and the thermal constraints used by Lellouch et al. (1989), McKay et al. (1989), Courtin et al. (1995), and Samuelson et al. (1997). Only with such work could the surface MMF and relative humidity be derived with confidence.

\section{ACKNOWLEDGMENTS}

Support of this work was provided by NASA through grant number GO07321.01-96A from Space Telescope Science Institute, which is operated by the Association of Universities for Research in Astronomy, Inc., under NASA Contract NAS5-26555. We greatly appreciated feedback from Chris McKay and an anonymous reviewer.

\section{REFERENCES}

Benner, D. C. 1979. The Visual and Near Infrared Spectrum of Methane and Its Application to Uranus, Neptune, Triton, and Pluto. Ph.D. dissertation, University of Arizona.

Combes, M., L. Vapillon, E. Gendron, A. Coustenis, O. Lai, R. Wittemberg, and R. Sirdey 1997. Spatially resolved images of Titan by means of adaptive optics. Icarus 129, 482-497.

Courtin, R., D. Gautier, and C. P. McKay 1995. Titan's thermal emission spectrum: Reanalysis of the Voyager infrared measurements. Icarus 114, 144-162.

Coustenis, A., E. Lellouch, J. P. Maillard, and C. P. McKay 1995. Titan's surface: composition and variability from the near infrared albedo. Icarus 118, 87-104.

Gibbard, S. G., B. Macintosh, B. Gavel, C. E. Max, I. de Pater, A. M. Ghez, E. F. Young, and C. P. McKay 1999. Titan: High resolution speckle images from the Keck telescope. Icarus 139, 189-201.

Goudfrooij, P., S. Baum, and J. R. Walsh 1997. Fringe correction for STIS nearIR long slit spectra using contemporaneous tungsten flat fields. In 1997 HST Calibration Workshop (S. Caertano, R. Jedrzejewski, and T. Keyes, Eds.), pp. 100-105. STScI, Baltimore.

Griffith, C. A. 1993. Evidence for surface heterogeneity on Titan. Nature 364, 511-514.

Griffith, C. A., T. Owen, and R. Wagener 1991. Titan's surface and troposphere, investigated with ground-based, near-infrared observations. Icarus 93, 362378.

Griffith, C. A., T. Owen, G. A. Miller, and T. Geballe 1998. Transient clouds in Titan's lower atmosphere. Nature 395, 575.
Griffith, C. A., J. L. Hall, and T. Geballe 2000. Detection of daily clouds on Titan. Science 290, 509-513.

Hansen, J. E., and L. D. Travis 1974. Light scattering in planetary atmospheres. Space Sci. Rev. 16, 527-610.

Hunten, D. M., M. G. Tomasko, F. M. Flasar, R. E. Samuelson, D. F. Strobel, and D. J. Stevenson 1984. Titan. In Saturn (T. Gehrels and M. S. Matthews, Eds.) pp. 671-759, Univ. of Arizona Press, Tucson.

Karkoschka, E., 1998. Methane, ammonia, and temperature measurements of the jovian planets and Titan from CCD-spectrophotometry. Icarus 133, 134-146.

Karkoschka, E., and Lorenz, R. D., 1997. Latitudinal variation of aerosol sizes inferred from Titan's shadow. Icarus 127, 369-379.

Khare, B. N., C. Sagan, E. T. Arakawa, F. Suits, T. A. Callcott, and M. W. Williams 1984. Optical constants of organic tholins produced in a simulated titanian atmosphere-From soft X-ray to microwave frequencies. Icarus $\mathbf{6 0}$, 127-137.

Kimble, R. A., and 88 colleagues, 1998. The on-orbit performance of the Space Telescope Imaging Spectrograph. Astrophys. J. 492, L83-93.

Kuiper, G. P. 1944. Titan: A satellite with an atmosphere. Astrophys. J. 100, 378-383.

Lellouch, E., A. Coustenis, D. Gautier, F. Raulin, N. Dubouloz, and C. Frère 1989. Titan's atmosphere and hypothesized ocean: A reanalysis of the Voyager 1 radio-occultation and IRIS $7.7 \mu \mathrm{m}$ data. Icarus 79, 328-349.

Lellouch, E., A. Coustenis, J.-P. Maillard, K. Strong, N. Deme, C. Griffith, and B. Schmitt 1992. The spectrum of Titan in the 1.06 and 1.28 micron windows. In Proc. Symp. Titan, ESA SP-338, pp. 353-358.

Lemmon, M. T. 1994. Properties of Titan's Haze and Surface. Ph.D. dissertation, University of Arizona.

Lemmon, M. T., E. Karkoschka, and M. Tomasko 1993. Titan's rotation: Surface feature observed. Icarus 103, 329-332.

Lemmon, M. T., E. Karkoschka, and M. Tomasko 1995. Titan's rotational lightcurve. Icarus 113, 27-38.

Lindal, G. F., G. E. Wood, H. B. Hotz, D. N. Sweetnam, V. R. Eshleman, and G. L. Tyler 1983. The atmosphere of Titan: An analysis of the Voyager 1 radio-occultation measurements. Icarus 53, 348-363.

Lorenz, R. D., M. T. Lemmon, P. H. Smith, and G. W. Lockwood 1999a. Rapid and complex seasonal change on Titan observed with the Hubble Space Telescope. Icarus 142, 391-401.

Lorenz, R. D., C. P. McKay and J. I. Lunine 1999b. Analytic investigation of climate stability on Titan: Sensitivity to volatile inventory. Planet. Space Sci. 47, 1503-1515.

Lutz, B. L., T. Owen, and R. D. Cess 1976. Laboratory band strengths of methane and their application to the atmospheres of Jupiter, Saturn, Uranus, Neptune, and Titan. Astrophys. J. 203, 541-551.

Lutz, B. L., T. O. Owen, and R. D. Cess 1982. Laboratory band strengths of methane and their application to the atmospheres of Jupiter, Saturn, Uranus, Neptune, and Titan. II. The red region 6000-7600 Angstroms. Astrophys. J. 258, 886-898.

McGrath, M. A., R. Courtin, T. E. Smith, P. D. Feldman, and D. F. Strobel 1998. The ultraviolet albedo of Titan. Icarus 131, 382-392.

McKay, C. P., J. B. Pollack, and R. Courtin 1989. The thermal structure of Titan's atmosphere. Icarus 80, 23-53.

McKay, C. P., S. C. Martin, C. A. Griffith, and R. M. Keller 1997. Temperature lapse rate and methane in Titan's troposphere. Icarus 129, 498-505.

McKay, C. P., A. Coustenis, R. E. Samuelson, M. Lemmon, R. Lorenz, M. Cabane, P. Rannou, and P. Drossart 2001. The physical properties of the organic aerosols and clouds on Titan. Planet. Space Sci. 49, 79-99.

Meier, R. M., B. A. Smith, T. C. Owen, and R. J. Terrile 2000. The surface of Titan from NICMOS observations with the Hubble Space Telescope. Icarus 145, 462-473.

Neckel, H., and D. Labs 1984. The solar radiation between 3300 and 12500 A. Solar Phys. 90, 205-258. 
Rannou, P., M. Cabane, E. Chassefière, R. Botet, and C. P. McKay 1995. Titan's geometric albedo: Role of the fractal structure of the aerosols. Icarus 111, 355-372.

Samuelson, R. E, N. R. Nath, and A. Borysow 1997. Gaseous abundances and methane supersaturation in Titan's troposphere. Planet. Space. Sci. 45, 959980.

Smith, P. H., M. T. Lemmon, R. D. Lorenz, L. A. Sromovsky, J. J. Caldwell, and M. D. Allison 1996. Titan's surface, revealed by HST imaging. Icarus 119, 336-349.

Strobel, D. F., M. E. Summers, and X. Zhu 1992. Titan's upper atmosphere: Structure and ultraviolet emissions. Icarus 100, 512-526.
Tomasko, M. G., and P. H. Smith 1982. Photometry and polarimetry of Titan: Pioneer 11 observations and their implications for aerosol properties. Icarus 51, 65-95.

Tomasko, M. G., M. T. Lemmon, L. R. Doose, P. H. Smith, A. Eibl, and R. A. West 1997. Models of the penetration of sunlight into the atmsophere of Titan. In Huygens: Science, Payload, and Mission, ESA SP-1177, pp. 345-358.

Trafton, L., 1975. The morphology of Titan's methane bands. I. Comparison with a reflecting layer model. Astrophys. J. 195, 805-814.

West, R. A., and P. H. Smith 1991. Evidence for aggregate particles in the atmospheres of Titan and Jupiter. Icarus 90, 330-333. 